\title{
ANGULAR DISTRIBUTIONS OF CHARGED PARTICLES FROM NUCLEAR REACTIONS
}

\author{
by P. M. ENDT, D. DE JONG*), M. BOGAARDT ${ }^{* *}$ ) \\ and B. KOUDIJS
}

Physisch Laboratorium der Rijksuniversiteit te Utrecht, Nederland

\section{Synopsis}

A description is given of equipment built for the measurement of angular distributions of charged particles released in nuclear reactions. Thin targets are bombarded by deuterons or protons accelerated by a cascadegenerator and analyzed by a magnet.

Secondary particles from the target are detected by means of photographic emulsions placed at $15^{\circ}$ intervals round the target. Results obtained with specific reactions (a.o. $\mathrm{Be}^{9}(d, p) \mathrm{Be}^{10}$ and $\mathrm{C}^{13}(d, p) \mathrm{C}^{14}$ ) will be dealt with in separate papers.

$\S 1$. Introduction. The accuracy with which reaction-energies ( $Q$-values) of charged particle reactions can be measured has been much improved in the last few years ${ }^{1}$ ). These measurements provide mass-differences between nuclei (if the final nucleus is left in the ground-state) or the positions of energy-levels in the final nucleus (if the final nucleus is left in an excited state). Other important data characterizing nuclear energy-states are their spin and parity but in general less is known about these quantities than about the positions of the states. Information about spin and parity can be obtained in many different ways one of which is the measurement of the angular distribution of particles released in nuclear reactions. This paper describes equipment built for this purpose. Measurements are confined to reactions in which both ingoing and outgoing particles are charged e.g. $(d, p)$ or $(d, \alpha)$ reactions.

Bombarding particles (generally deuterons) are produced in a magnetic ion-source and accelerated by the Utrecht cascadegenerator. After magnetic analysis they hit thin targets prepared by evaporation in vacuum onto thin aluminium backings. The reaction

*) Now at N.V. Nederlandse Instrumenten en Electrische Apparaten Fabriek , NIEAF” te Utrecht, Nederland.

**) Now at Institutt for Atomenergi, Kjeller, Norge. 
products (protons, alpha-particles or tritons) are detected by means of nuclear emulsions placed at $15^{\circ}$ intervals round the target $(\S 2)$. The number of tracks per unit area in these emulsions is counted under a microscope from which the relative angular distribution is obtained after several small corrections have been applied $(\S 3)$. Relative angular distributions can be converted into absolute differential cross-sections by measuring the total charge collected on the target and the thickness of the target (§ 4).

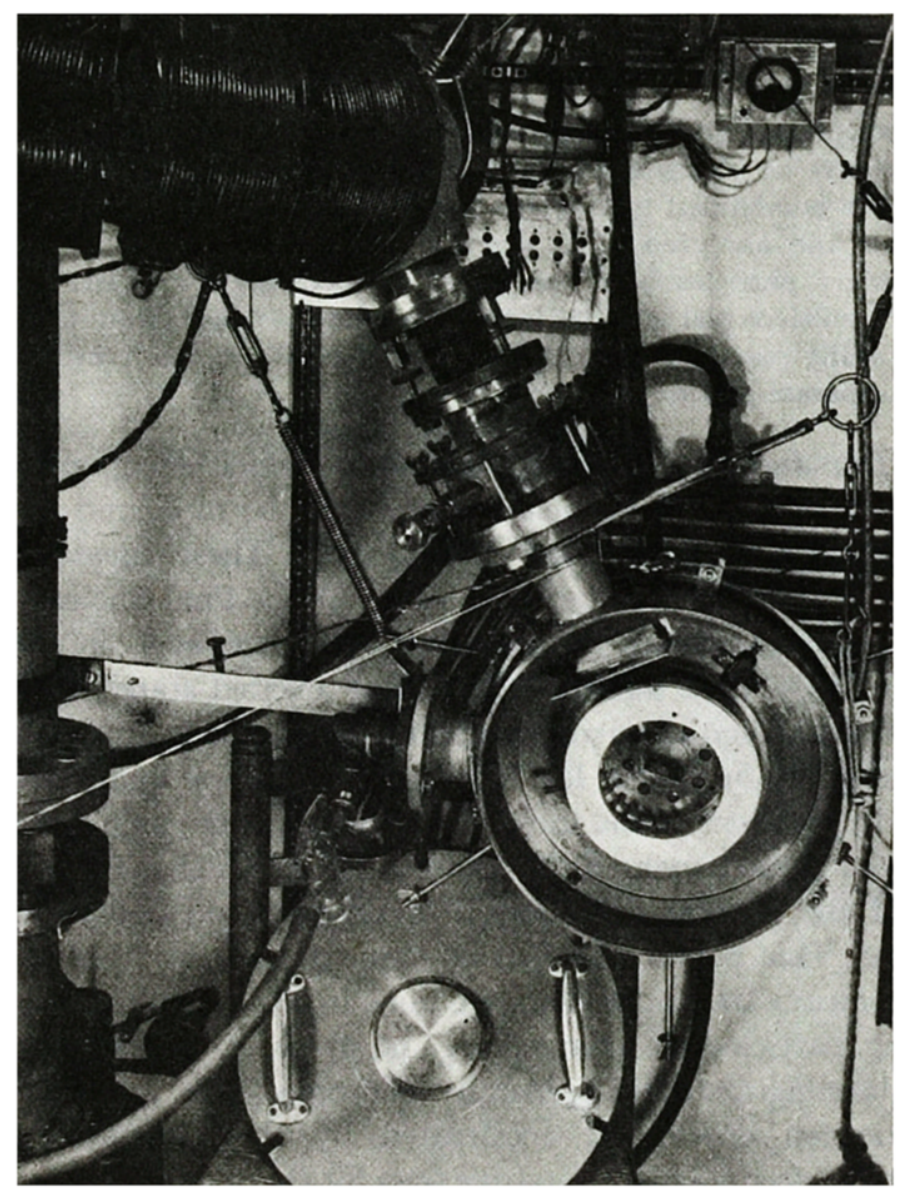

Fig. 1. Analyzing magnet and targetbox. The lid of the targetbox has been removed. Inside the targetbox can be seen the plateholder, containing target and nuclear emulsions. The lid of the plateholder, on which are fixed the slit system and the aluminium foil round the target, has also been removed. 
§2. Experimental equipment. Cascade-generator, acceleration tube and ion-source are described in detail in the preceding paper by $\mathrm{V}$ e en stra e.a. ${ }^{2}$ ). A photograph of some of the equipment assembled in the targetroom is given in Fig. 1. It shows the $30^{\circ}$ analyzing magnet and the round iron box containing target and nuclear emulsions.

Fifteen nuclear emulsions $\left(1^{\prime \prime} \times 1 \frac{1}{3}^{\prime \prime}\right)$ are placed round the target at $15^{\circ}$ intervals in a cylindrical aluminium plate-holder as shown in Fig. 2.

To avoid shadow effects from the brass frame holding the target-

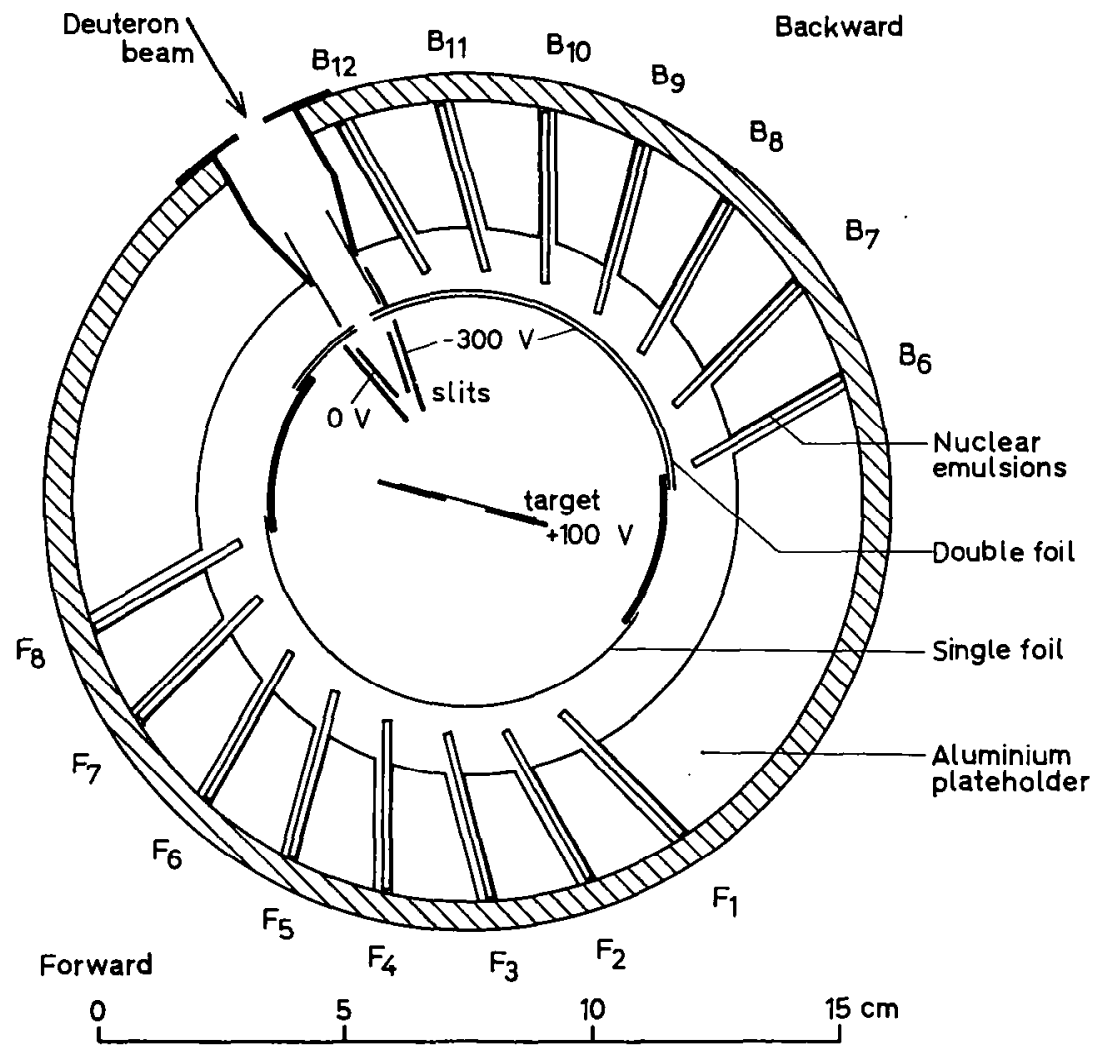

Fig. 2. Plate-holder. Fifteen nuclear emulsions are placed at $15^{\circ}$ intervals round the target. The outer slitsystem (at $-300 \mathrm{~V}$ ) serves to prevent secondary electrons produced at the edges of the grounded inner slit (width $1.5 \mathrm{~mm}$ ) from reaching the target. The aluminium foil $(7 \mu)$ round the target prevents blackening of the nuclear plates by elastically scattered deuterons. 
foil, the target is placed at $45^{\circ}$ to the bombarding beam; outgoing particles making angles from $0^{\circ}$ to $105^{\circ}$ with the direction of the beam are detected by plates numbered $F_{1}$ through $F_{8}$ while plates $\mathrm{B}_{6}$ through $\mathrm{B}_{12}$ detect particles making angles from $75^{\circ}$ to $165^{\circ}$ with the beam. The average distance from target to plates is $60 \mathrm{~mm}$ and the average angle of incidence of particles on the surface of the emulsions is $15^{\circ}$. It is seen that the angles of deflection of $75^{\circ}$, $90^{\circ}$ and $105^{\circ}$ are each covered by two plates. This is done to be able to detect and to correct for any eccentricity of the spot where the beam hits the target. This eccentricity is never very large however (see also $\S 3$ ) because the beam is defined by a slit of only 1.5 $\mathrm{mm}$ width placed just in front of the target (distance slit - target is $25 \mathrm{~mm}$ ). The height of the slit is $12 \mathrm{~mm}$ but the beam diameter is here much smaller, about $5 \mathrm{~mm}$, due to the focussing action of the electric lenses in the acceleration tube.

By optical means this slit was accurately fixed in position in the median plane of the plate-holder. By the same optical arrangement the surface of the target was brought in a plane through the axis of the plate-holder.

After a preliminary beam exposure the position of the burning mark on a target was determined optically and then the plateholder was adjusted so as to make the beam hit the center of the target.

To prevent blackening of the plates by elastically scattered primary particles the plates are shielded by a $7 \mu$ aluminium foil held in position by a cylindrical brass frame. It was found impossible to obtain thin foils quite free from small pinholes. To make up for these defects the foil screening plates $B_{6}$ through $B_{12}$ was taken double. This is not necessary for plates $F_{1}$ through $F_{8}$ because in these directions a second screen is provided by the $7 \mu$ aluminium target backing. Of course these screens limit the scope of our measurements to reactions where the range of secondary particles in aluminium is at least $14 \mu$, but this is not found a severe handicap because short range groups are mostly obscured anyhow by intensive contaminant groups (e.g. from $0^{16}(d, \alpha) \mathrm{N}^{14}$ and $0^{16}(d, p) 0^{17 *}$ ).

For determinations of absolute differential cross-sections (see also $\S 4$ ) it is necessary to measure the total charge collected on the target during an exposure, free from errors introduced by secondary electrons. To this end the plate-holder (and also the aluminium foil 
surrounding the target), insulated from the target-box, is given a bias potential of $-300 \mathrm{~V}$ in respect to the grounded target-box. The target, insulated from target-box and plate-holder, is connected to a current-integrator. The average target potential is $+100 \mathrm{~V}$. The V-shaped beam entrance slit is grounded. To prevent secondary electrons produced at the edges of the slit from reaching the target, a second slit of $2.5 \mathrm{~mm}$ width at a potential of $-300 \mathrm{~V}$ is mounted between the first slit and the target. If the bias voltage on plate-holder and second slit is lowered from $0 \mathrm{~V}$ to $-300 \mathrm{~V}$, the target current first decreases fast to reach a constant value below $-150 \mathrm{~V}$, indicating that errors from secondary electrons are probably well eliminated.

The current-integrator is built after a design given by $\mathrm{E} 1 \mathrm{~m}$ or e and $\mathrm{S} \mathrm{a} \mathrm{n} \mathrm{d} \mathrm{s}{ }^{3}$ ). The pulses at the output of the current-integrator, each corresponding to 0.05 microcoulomb, are counted with a standard scaling circuit. The target current is also read by a galvanometer, carefully insulated from ground to avoid leakage currents.

The high voltage of the cascade-generator is measured with a resistor column (1200 resistors of 1 Megohm each) and mA-meter. Both were calibrated with an error smaller than $\frac{1}{2} \%$. Although the resistor is put in oil its resistance shows a slow drift due to heat development after the generator is switched on. To prevent a corresponding drift in high voltage during long exposures the current through the coils of the analyzing magnet is kept constant rather than the reading of the high voltage $\mathrm{mA}$-meter. The variac controlling the high-voltage is varied manually in such a way as to keep the beam coming through the slit system in the target-box regardless of the reading of the mA-meter.

§3. Processing of nuclear emulsions and counting of tracks. Throughout this work Ilford C2 emulsions are used of $50 \mu, 100 \mu$ or $200 \mu$ thickness, the thickness depending on the maximum range to be expected for the secondary particles from a particular target. To obtain homogeneous development throughout the emulsion "temperature development" is applied as recommended by Dil wor th, Occhialini and Payne ${ }^{4}$.

Two binocular microscopes, one Leitz and one Zeiss, are in use for the scanning of the plates. Total magnification is adapted to the particular tracklength of particles being counted. The magnif- 
ication of a binocular microscope depends also on the eye-base used which can introduce errors if different observers assist in the counting of one angular distribution. For observers with widely different eye-bases this can cause differences in magnification of up to $3 \%$. The Zeiss microscope allows to compensate for this effect as both eyepieces can be screwed higher or lower in the tubus such as to keep the total magnification constant for different eye-bases.

Before the angular distribution of any specific reaction can be measured, one has to be certain that the following two conditions are met:

a) the tracklength of the particular particle group has to be well different from that of other groups originating from the target element or from any contamination on the target;

b) to allow fast counting the track density in the emulsion has to be neither too small nor too large. A good average is between 100 and 1000 tracks per square millimeter.

The fulfilment of these conditions is investigated by means of a range analysis obtained by measuring the range of all tracks on a certain area of the emulsion. The assignment of the distinct particle groups each corresponding to a maximum in this range spectrum is done by comparing the measured range maxima with the ranges to be expected for a number of probable reactions. Ranges are calculated by computing the energy of the outgoing particle (from $Q$-value, energy of the bombarding particle and angle between inand outgoing particle ${ }^{5}$ ) and using the range-energy relation valid for the emulsion ${ }^{6}$ ). These often recurring computations can be considerably simplified by making use of a suitable nomogram. Finally corrections are applied to the calculated range for energy loss in the target, the target backing and the aluminium screen, and for the dip in the emulsion which shortens all tracks as seen under the microscope by about $3 \%$. Additional proof for the correctness of any particular assignment can be obtained by comparing measured and calculated ranges not only at one angle but at several widely different angles e.g. $0^{\circ}, 90^{\circ}$ and $165^{\circ}$. The variation of the range as a function of angle is generally different for different reactions.

It has not been tried to distinguish the tracks of different particles (e.g. protons, tritons and alpha-particles) by difference in grain density or scattering in the emulsion. These differences 
are too small to be of much use if many thousands of tracks are to be counted in a reasonably short time.

After a range analysis has been made the counting can start of tracks belonging to one particular particle group. All tracks are counted having a range between an upper and a lower limit which are deduced from the measured range analysis taking into account the width of the group and the range difference with neighbouring groups. These limits are of course different from plate to plate because the energy of outgoing particles is dependent on angle.

The actual counting is done on a mechanical register operated by a pedal which leaves both hands free for manipulation with the microscope. Under optimum conditions a skilled observer can count about 1000 tracks per hour. To obtain the same relative statistical error $(3 \%)$ for all points of an angular distribution generally about 1000 tracks are counted per plate making the total number of tracks counted for one angular distribution about 15,000. Only tracks are counted of particles evidently originating from the target, incident on the plate in the right direction and at the right dip angle. Thus nearly all "background" e.g. particles scattered by the plate-holder can be eliminated.

To the track densities thus measured several small corrections have to be applied to obtain the angular distribution. The distance of the area counted on a plate to the target may differ by 1 or $2 \mathrm{~mm}$ from the average distance $(60 \mathrm{~mm})$ for which difference has to be corrected. After careful alignment of target-box and slit system (see $\S 2$ ) the eccentricity of the beam spot on the target is now less than $0.2 \mathrm{~mm}$. Corrections due to this eccentricity can be neglected.

Finally the angular distribution measured in the laboratory system has to be transferred to the center of mass system. The transformation formulae for intensities and angles can easily be derived. They are given e.g. by $\mathrm{H}$ e y d e $\mathrm{n} \mathrm{burg}$ and I $\mathrm{ng} l$ is ${ }^{7}$ ).

§4. Differential cross-sections. From relative angular distributions absolutc differential cross-sections may be obtained by the additional measurement of the total charge collected on the target during the exposure and of the target thickness in grams per square centimeter. The former is measured by the current-integrator, the latter can be determined by weighing. Whereas the error in the number of secondary particles per steradian is certainly 
smaller than $5 \%$ (the statistical error is only $3 \%$ ), the errors involved in the measurement of total charge and target thickness are much larger. The deuteron beam may contain an unknown amount of normal hydrogen molecular ions, the target may not be of quite uniform thickness etc. An estimate of these errors gives an over-all accuracy of absolute cross-sections of about $30 \%$.

Acknowledgements. This work is part of the research program of the Stichting voor Fundamenteel Onderzoek der Materie, which was made possible by a subvention from the Stichting voor Zuiver Wetenschappelijk Onderzoek.

The authors are indebted to Prof. J. M. W. Milat $z$ for his stimulating interest, to P. C. V e e n $\mathrm{t} r$ a for his highly valued preparatory work regarding cascade-generator and ion-source, to A. J. L. v a n E g m o n d, who supervised the construction of the current-integrator in the electronics department of this laboratory, to H. M. Jongerius, C. H. Paris and F. P. G. $\mathrm{V} \mathrm{a} \mathrm{l} \mathrm{c} \mathrm{kx}$, who assisted with the construction and try-out of most of the equipment, and to A. N. va n S $\mathrm{r}$ a t e $\mathrm{n}$ for his accurate machining of target-box and plate-holder.

Received 21-3-52.

1) Hornyak, Lauritsen, Morrison and Fowler, Rev. mod. Phys. 22: (1950) 291; Strait, Van Patter, Buechner and Sperduto, Phys. Rev. 81 (1951) 747.

2) Veenstra, Jongerius, Paris and Valckx, Physica 18 (1952) 378.

3) E $1 \mathrm{~m}$ ore, W. C. and S ands, M., Electronics, New York, Mc Graw Hill, 1949.

4) Dilworth, Occhialini and Payne, Nature 162 (1948) 102.

5) Livingston, M. S. and Bet he, H. A., Rev. mod. Phys. 9 (1937) 245.

6) Boga ardt, M. et Vigneron, L., J. Phys. Radium 11 (1950) 652; R otb l a t, J, Nature 167 (1951) 550.

7) Heydenburg, N. P. H. and Inglis, D. R., Phys. Rev. 73 (1948) 230 and Phys. Rev. 71 (1948) 406. 